\title{
wrasousus
}

\section{Altered neuronal circuits control metabolic fate}

Researchers from Germany and the USA have pinpointed a critical developmental window during pregnancy when a mother's nutritional habits have the greatest effect on the future metabolic health of her offspring. Using a mouse model, they found that mothers fed a high-fat diet during lactation exerted pronounced metabolic effects upon their progeny owing to abnormal neuronal connections within the hypothalamus.

Epidemiological studies have conclusively established that altered maternal metabolism during pregnancy can have long-lasting consequences for offspring, predisposing them to obesity and diabetes mellitus later in life (so-called 'metabolic programming'). As the mechanisms underlying this phenomenon were largely unknown, the research group generated an experimental model of metabolic programming by feeding mice either normal chow or a high-fat diet at various time points during and after pregnancy.

The investigators found that the metabolic programming associated with maternal high-fat feeding during lactation was caused by disruption of projections from proopiomelanocortin (POMC) and agouti-related peptide neurons of the melanocortin circuitry to their target sites within the hypothalamus. Deletion of the insulin receptor from POMC neurons in the offspring restored normal development of POMC neuronal projections to the pre-autonomic compartment of the paraventricular nucleus of the hypothalamus. This finding suggested that elevated insulin levels in the offspring in response to a maternal high-fat diet could contribute to the observed suppression of neuronal projection formation.

The next step for the research group is to determine the status of other brain networks following metabolic programming. "We are currently investigating whether a maternal high-fat diet has similar effects on other neuronal circuits, which might explain behavioural alterations observed in the offspring of metabolically challenged mothers," states co-lead investigator Jens Brüning (Max Planck Institute for Neurological Research, Cologne).

The researchers point out that any attempt to extrapolate data gathered from mouse studies to the human situation should be approached with caution. Nevertheless, the formation of neuronal circuit projections, which occurs during lactation in mice, takes place in the third trimester of human pregnancy, a period when the mother is particularly prone to gestational diabetes mellitus and excessive weight gain.

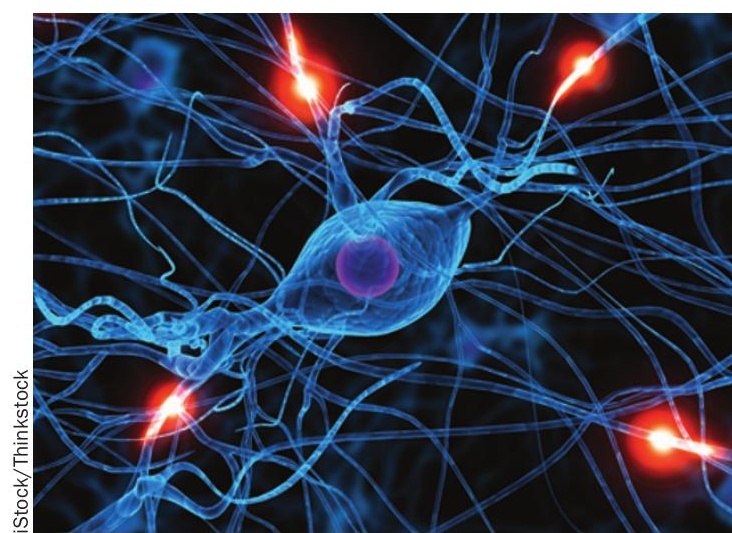

"Our study indicates that alterations of maternal glucose metabolism during this phase might have long-lasting effects on the metabolic fate of the unborn child through altering the architecture of key hypothalamic centres involved in the control of energy and glucose homeostasis," says Brüning. "Close attention should, therefore, be paid to maintaining tight glycaemic control during the third trimester of pregnancy."

Vicky Heath

Original article Vogt, M. C. et al. Neonatal insulin action impairs hypothalamic neurocircuit formation in response to maternal high-fat feeding. Cell doi:10.1016/ j.cell.2014.01.008 